\title{
Enhancing triplet superconductivity by the proximity to a singlet superconductor in oxide heterostructures
}

\author{
Mats Horsdal, ${ }^{1,2}$ Giniyat Khaliullin, ${ }^{3}$ Timo Hyart, ${ }^{4,5}$ and Bernd Rosenow ${ }^{2}$ \\ ${ }^{1}$ Department of Physics, University of Oslo, P. O. Box 1048 Blindern, N-0316 Oslo, Norway \\ ${ }^{2}$ Institut für Theoretische Physik, Universität Leipzig, D-04009 Leipzig, Germany \\ ${ }^{3}$ Max Planck Institute for Solid State Research, Heisenbergstrasse 1, D-70569 Stuttgart, Germany \\ ${ }^{4}$ Department of Physics and Nanoscience Center, University of Jyväskylä, P.O. Box 35 (YFL), FI-40014 University of Jyväskylä, Finland \\ ${ }^{5}$ Instituut-Lorentz, Universiteit Leiden, P.O. Box 9506, 2300 RA Leiden, The Netherlands \\ (Received 19 January 2015; revised manuscript received 25 April 2016; published 6 June 2016)
}

\begin{abstract}
We show how in principle a coherent coupling between two superconductors of opposite parity can be realized in a three-layer oxide heterostructure. Due to strong intraionic spin-orbit coupling in the middle layer, singlet Cooper pairs are converted into triplet ones and vice versa. This results in a large enhancement of the triplet superconductivity, persisting well above the native triplet critical temperature.
\end{abstract}

DOI: 10.1103/PhysRevB.93.220502

The prospect of realizing Majorana bound states that can be used for quantum information processing has led to a large interest in odd-parity superconductivity. Native triplet superconductivity, believed to be realized in, e.g., $\mathrm{Sr}_{2} \mathrm{RuO}_{4}$, is fragile and only present at very low temperatures [1]. It is known that a singlet superconductor (SC) can induce triplet pairing correlations in systems with Rashba spinorbit coupling and/or ferromagnetism due to the proximity effect [2-10], and it has been suggested to induce triplet superconductivity in hybrid structures with such properties [11-14]. Rashba spin-orbit coupling and ferromagnetism can also give rise to a Josephson coupling between $s$ - and $p$-wave SCs $[15,16]$.

Here, we suggest an alternative way to improve the robustness of an odd-parity SC by tunnel coupling it to an even-parity singlet SC in all-oxide-based heterostructures. This mechanism is neither due to Rashba coupling nor ferromagnetism, but by virtue of a strong intraionic spin-orbit coupling inherent to late transition-metal compounds such as iridium oxide $\mathrm{Sr}_{2} \mathrm{IrO}_{4}$ [17]. To have a coherent coupling between two SCs of opposite parity, the tunneling has to "rotate" the Cooper pairs, since the wave functions of the oddand even-parity superconducting condensates are orthogonal to each other. We consider a heterostructure consisting of three quasi-two-dimensional layers: The even-parity spinsinglet "cuprate" SC ( $B$ layer) is separated from the oddparity spin-triplet "ruthenate" SC ( $A$ layer) by an insulating layer, the "iridate convertor" (see the inset of Fig. 1). The superconductivity takes place in the $d_{x^{2}-y^{2}}$ band of the $B$ layer, and in the Ru orbitals of $t_{2 g}$ symmetry in the $A$ layer. The tunnel coupling between the two SCs is provided via the spin-orbit entangled Ir $t_{2 g}$ orbitals in the middle layer. We will show that a strong intraionic spin-orbit coupling in the middle layer gives rise to an effective tunneling matrix between the two SCs, where the diagonal and off-diagonal elements have opposite parity. The time-reversal and mirror-symmetric tunneling matrix results in a coherent coupling between the two SCs and leads to an enhancement of the odd-parity order parameter (see Fig. 1).

This Rapid Communication is organized as follows: We start by describing the model consisting of two SCs with opposite parity and show, on a phenomenological level, that a general time-reversal and reflection-symmetric tunneling can give rise to coherent coupling of the order parameters of different parity in the two layers. We then discuss experimental signatures of such a coupling, that is, a dramatic enhancement of the triplet order parameter persisting well above the "native" critical temperature of the triplet SC. Finally, we show how the electron tunneling with the desired properties can be realized in oxide heterostructures, by considering a specific example of microscopic tunneling processes via the spin-orbit entangled Ir $t_{2 g}$ orbitals in the middle layer.

Model. The Hamiltonian of the system $H=H_{A}+H_{B}+$ $H_{A B}$ comprises the Hamiltonians $H_{A / B}$ for the odd-parity spin-triplet $A$ layer and the even-parity spin-singlet $B$ layer, respectively, and the effective tunneling term $H_{A B}$ due to the iridate convertor between these layers. The Hamiltonian for the triplet $\mathrm{SC}$ reads as $H_{A}=\frac{1}{2} \sum_{\mathbf{k}} \bar{A}_{\mathbf{k}} \bar{H}_{A}(\mathbf{k}) A_{\mathbf{k}}$, where the electron fields have been written in Nambu form, $\bar{A}_{\mathbf{k}}=$ $\left(\bar{a}_{\mathbf{k} \uparrow}, \bar{a}_{\mathbf{k} \downarrow}, a_{-\mathbf{k} \uparrow}, a_{-\mathbf{k} \downarrow}\right)$, and

$$
\bar{H}_{A}(\mathbf{k})=\left(\begin{array}{cc}
\xi_{A}(\mathbf{k}) & \hat{\Delta}_{A}(\mathbf{k}) \\
\hat{\Delta}_{A}^{\dagger}(\mathbf{k}) & -\xi_{A}(\mathbf{k})
\end{array}\right) .
$$

It is assumed that the dispersion $\xi_{A}(\mathbf{k})$ is independent of spin. The order parameter matrix $\hat{\Delta}_{A}(\mathbf{k})=i \mathbf{d}_{\mathbf{k}} \cdot \sigma \sigma_{y}$, where $\sigma_{j}(j=$ $x, y, z)$ are the Pauli matrices.

The singlet-layer Hamiltonian $H_{B}$ takes a form similar to $H_{A}$, except for the replacements $\xi_{A}(\mathbf{k}) \rightarrow \xi_{B}(\mathbf{k})$, $\hat{\Delta}_{A}(\mathbf{k}) \rightarrow \hat{\Delta}_{B}(\mathbf{k})$ with $\hat{\Delta}_{B}(\mathbf{k})=i \sigma_{y} \Delta_{B \mathbf{k}}$, and $\bar{A}_{\mathbf{k}} \rightarrow \bar{B}_{\mathbf{k}}=$ $\left(\bar{b}_{\mathbf{k} \uparrow}, \bar{b}_{\mathbf{k} \downarrow}, b_{-\mathbf{k} \uparrow}, b_{-\mathbf{k} \downarrow}\right)$.

A general tunneling term between the $A$ and $B$ layers can be written as $H_{A B}=\frac{1}{2} \sum_{\mathbf{k}} \bar{A}_{\mathbf{k}} T(\mathbf{k}) B_{\mathbf{k}}+$ H.c., where

$$
T(\mathbf{k})=\left(\begin{array}{cc}
\hat{T}(\mathbf{k}) & 0 \\
0 & -\hat{T}^{*}(-\mathbf{k})
\end{array}\right), \quad \hat{T}(\mathbf{k})=\left(\begin{array}{cc}
P_{\mathbf{k}} & R_{\mathbf{k}} \\
S_{\mathbf{k}} & Q_{\mathbf{k}}
\end{array}\right) .
$$

Time-reversal invariance of the Hamiltonian gives the following restriction on the elements of the tunneling matrix: $P_{\mathbf{k}}=Q_{-\mathbf{k}}^{*}$ and $R_{\mathbf{k}}=-S_{-\mathbf{k}}^{*}$. The system under consideration is invariant under reflections about the $x z$ plane, $\mathcal{M}_{x}$, where the position and spin transform as $(x, y) \rightarrow(x,-y)$ and $\left(S_{x}, S_{y}, S_{z}\right) \rightarrow\left(-S_{x}, S_{y},-S_{z}\right)$. There is a similar symmetry under reflection about the $y z$ plane, $\mathcal{M}_{y}$, so that in the spin sector $\mathcal{M}_{x}$ and $\mathcal{M}_{y}$ correspond to $i \sigma_{y}$ and $i \sigma_{x}$, respectively. 


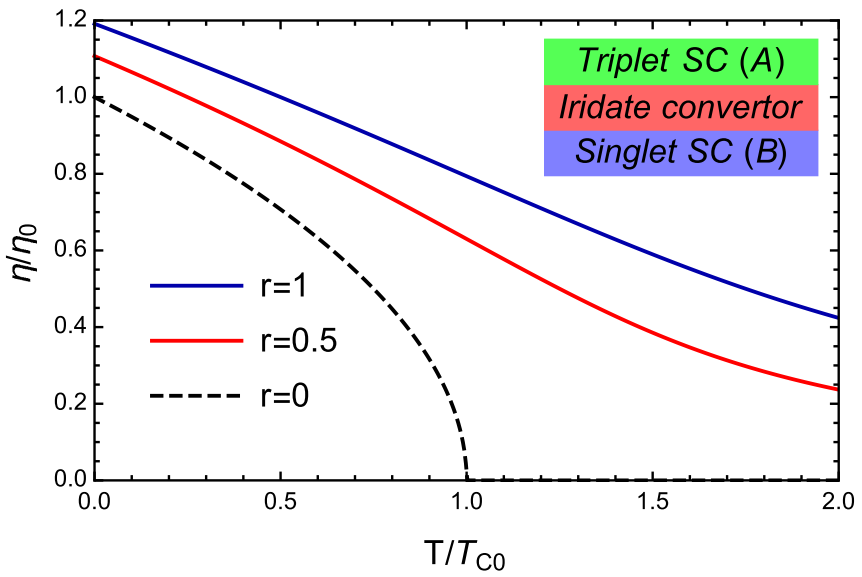

FIG. 1. The $p$-wave order parameter $\eta$ as a function of temperature for different values of the enhancement parameter $r$ [defined below in Eq. (5)]. ( $\eta_{0}$ and $T_{C 0}$ are bare values of the order parameter and critical temperature.) The inset shows the three-layer hybrid structure: a singlet SC $(B)$, the "iridate convertor," and the triplet $\mathrm{SC}(A)$.

Since a spin-orbit coupling $\mathbf{L} \cdot \mathbf{S}$ is invariant under these symmetries, the tunnel Hamiltonian $\hat{T}$ is invariant under the combined operation $\mathcal{M}_{x} \mathcal{M}_{y}$ and obeys $\sigma_{z} \hat{T}(-\mathbf{k}) \sigma_{z}=\hat{T}(\mathbf{k})$. For this reason, $P$ and $Q$ are even, $P_{-\mathbf{k}}=P_{\mathbf{k}}$ and $Q_{-\mathbf{k}}=Q_{\mathbf{k}}$, and $R$ and $S$ are odd, $R_{-\mathbf{k}}=-R_{\mathbf{k}}$ and $S_{-\mathbf{k}}=-S_{\mathbf{k}}$ [18].

The free energy for the system can be calculated by integrating out the fermionic degrees of freedom [20]. It takes the form $F=F_{A}+F_{B}+F_{A B}$, where $F_{A(B)}$ is the free energy for the $A(B)$ layer and $F_{A B}$ is the coupling between the two layers. Our focus is on the coupling between the two SCs. Assuming time-reversal invariance of the system and unitary $p$-wave superconductivity in the $A$ layer, the coupling to second order in $H_{A B}$ is [2]

$$
\begin{aligned}
F_{A B} \simeq & \frac{1}{2} \sum_{\mathbf{k}} W_{\mathbf{k}} \Delta_{B \mathbf{k}}^{*}\left\{d_{z \mathbf{k}}\left(\left|P_{-\mathbf{k}}\right|^{2}+\left|R_{-\mathbf{k}}\right|^{2}\right)\right. \\
& \left.+\left(d_{x \mathbf{k}}+i d_{y \mathbf{k}}\right) P_{\mathbf{k}} R_{-\mathbf{k}}+\left(d_{x \mathbf{k}}-i d_{y \mathbf{k}}\right) P_{\mathbf{k}}^{*} R_{-\mathbf{k}}^{*}\right\}+ \text { H.c. },
\end{aligned}
$$

where only the terms sensitive to the phase difference between the two layers have been kept [21]. Here, $W_{\mathbf{k}}=\left(\Phi_{\mathbf{k}}^{A}-\Phi_{\mathbf{k}}^{B}\right) /\left(E_{B \mathbf{k}}^{2}-E_{A \mathbf{k}}^{2}\right)$, a function $\Phi_{\mathbf{k}}^{A / B}=$ $\frac{1}{E_{A / B}} \tanh \frac{\beta E_{A / B}}{2}$, and $\beta$ is the inverse temperature. The quasiparticle energies are given by $E_{A / B}(\mathbf{k})=\sqrt{\xi_{A / B}^{2}(\mathbf{k})+\left|\Delta_{A / B}\right|^{2}}$. The symmetry $\mathcal{M}_{x} \mathcal{M}_{y}$ corresponds to $\mathbf{k} \rightarrow-\mathbf{k}$ and a $\pi$ rotation of spins around the $z$ axis, such that the invariance of the $\mathbf{d}_{\mathbf{k}}$ vector implies $d_{x / y-\mathbf{k}}=-d_{x / y \mathbf{k}}$ and $d_{z-\mathbf{k}}=d_{z \mathbf{k}}$, and as a consequence, $d_{z \mathbf{k}} \equiv 0$. In Eq. (3), this is reflected by the fact that $d_{x / y \mathbf{k}}\left(d_{z \mathbf{k}}\right)$ is multiplied by an odd (even) function of $\mathbf{k}$, so only $d_{x / y \mathbf{k}}$ terms may couple to a $\mathbf{k}$-even $\Delta_{B \mathbf{k}}$.

To illustrate the effect with a simple toy model, we assume that the elements of the tunneling matrix take the simple form $P_{\mathbf{k}}=i P$ and $R_{\mathbf{k}}=R\left(\sin k_{x}-i \sin k_{y}\right)$, with $P$ and $R$ real. To get an idea which combinations of order parameters in the singlet and triplet layers give a nonvanishing coupling, we consider the following order parameters for the triplet layer [2],

$$
\begin{aligned}
& \Gamma_{1 / 3}^{-}: \mathbf{d}_{\mathbf{k}}=\eta e^{i \theta}\left(\mathbf{e}_{x} \sin k_{x} \pm \mathbf{e}_{y} \sin k_{y}\right), \\
& \Gamma_{2 / 4}^{-}: \mathbf{d}_{\mathbf{k}}=\eta e^{i \theta}\left(\mathbf{e}_{x} \sin k_{y} \mp \mathbf{e}_{y} \sin k_{x}\right),
\end{aligned}
$$

and that the singlet-layer pairing has either $s$ - or $d$-wave symmetry, $\Delta_{B \mathbf{k}}^{s}=\Delta_{0}\left(\cos k_{x}+\cos k_{y}\right)$ and $\Delta_{B \mathbf{k}}^{d}=\Delta_{0}\left(\cos k_{x}-\right.$ $\cos k_{y}$ ), respectively. Here $\eta, \Delta_{0}>0$, and $\theta$ is the phase difference between the $p$ - and $s / d$-wave order parameters. For this model, except for the cases $\Gamma_{2}^{-}$and $s$ wave or $\Gamma_{4}^{-}$and $d$ wave, the integrand in Eq. (3) is either exactly vanishing, antisymmetric under mirroring about the $y$ axis, or antisymmetric under a $90^{\circ}$ rotation about the origin. Therefore only the combinations $\left(\Delta_{B \mathbf{k}}^{s}, \Gamma_{2}^{-}\right)$and $\left(\Delta_{B \mathbf{k}}^{d}, \Gamma_{4}^{-}\right)$ give a nonvanishing $F_{A B}$. The first combination constitutes a fully gapped helical topologically nontrivial SC [23-25].

Proximity enhancement. The coupling between the two SCs leads to a dramatic enhancement of the triplet order parameter, as we will see now. Close to the native critical temperature $T_{C 0}$ of the $A$ layer, the triplet order parameter is small and the free energy can be expanded in $\eta$. Assuming that the singlet pairing in the $B$ layer is robust, $\Delta_{0} \gg T_{C 0}$, and its variation near $T_{C 0}$ is negligible, we can ignore the $F_{B}$ term since it only contributes a constant to the energy. To fourth order in $\eta$, the free energy can then be written as

$$
F=(t-1) a \eta^{2}+\frac{1}{2} b \eta^{4}-r a \eta_{0} \eta \cos \theta,
$$

where $a, b>0$ are constants describing the native $A$ layer [26,27], and $t=T / T_{C 0}$. The enhancement factor $r$ is defined by $\frac{F_{A B}}{a \eta_{0}}=-r \eta \cos \theta$. Here, $\eta_{0}$ is the zero temperature gap at vanishing $F_{A B}$. For fixed $\eta$, it is clear that $F$ is minimized when $\cos \theta=\operatorname{sgn}(r)$. The value attained by the $p$-wave order parameter is found by minimizing $F$ with respect to $\eta$. Figure 1 shows $\eta$ as a function of temperature for representative values of $r$ (see the discussion below). We see that the coupling to the $B$ layer can give a large enhancement of the triplet superconductivity persisting well above $T_{C 0}$.

Due to the amplification of the triplet order parameter, the anomalous pair-tunneling current will persist also above the "native" critical temperature $T_{C 0}$ in the form of a zero-bias peak in the differential conductance [28]. More directly, the proximity-enhanced triplet gap (proportional to $\eta$ ) and its temperature dependence (see Fig. 1) can be probed by scanning tunneling microscopy (STM) or angle-resolved photoemission spectroscopy (ARPES) experiments.

Iridate convertor. We now discuss a possible realization of the layered structure that gives rise to a tunneling matrix of the form (2), where the diagonal elements have even parity and the off-diagonal ones have odd parity, providing a coherent coupling between the $A$ and $B$ SCs.

We assume that all three layers have a square lattice geometry with similar lattice constants. A possible candidate, which can be designed by a modern layer-by-layer growth technique [29], could be the oxide heterostructure $\mathrm{Sr}_{2} \mathrm{RuO}_{4} / \mathrm{Sr}_{2} \mathrm{IrO}_{4} / \mathrm{La}_{2} \mathrm{CuO}_{4}$. The pairing in the $B$ (cuprate) layer takes place in the $\mathrm{Cu} d_{x^{2}-y^{2}}$ orbitals designated by the annihilation operator $b_{\mathbf{r} \sigma}$, where $\mathbf{r}$ is the (two-dimensional) lattice position and $\sigma$ the spin, while the pairing in the $A$ (ruthenate) layer is assumed to take place in the $\mathrm{Ru} d_{x y}$ orbitals [30], labeled by $a_{\mathbf{r} \sigma}$. The relevant orbitals in the middle layer 
(a)

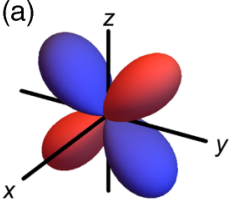

(d)

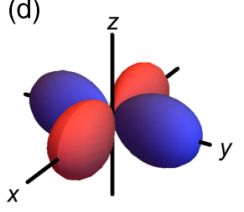

(b)

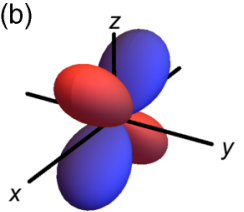

(c)

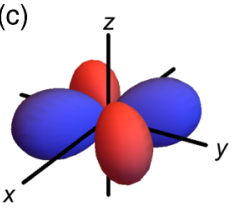

FIG. 2. The shape of the atomic orbitals involved: (a), (b) $d_{y z}$ and $d_{x z}$ orbitals for the iridate convertor, (c) $d_{x y}$ orbital for the iridate convertor and $A$ layer, and (d) $d_{x^{2}-y^{2}}$ orbital for the $B$ layer. (e) The hopping paths (green lines) between the $B$ layer (bottom) and the iridate convertor layer (top). Similar paths apply to the hopping between the iridate convertor layer and the $A$ layer.

are the Ir $t_{2 g}$ orbitals $d_{y z}, d_{x z}, d_{x y}$ denoted below by $\alpha_{\mathbf{r} \sigma}, \beta_{\mathbf{r} \sigma}$, $\gamma_{\mathbf{r} \sigma}$, respectively. Figure 2 shows the orientation of the above orbitals.

We consider first the tunneling between the $B$ layer and the middle layer. Figure 2 shows the possible hopping paths between the two layers. There can be no hopping between a $d_{x^{2}-y^{2}}$ orbital located at $\mathbf{r}$ and a $d_{x z}$ orbital located at $\mathbf{r} \pm \mathbf{e}_{y}+$ $\mathbf{e}_{z}$ for symmetry reasons [35]. On the other hand, hopping from a $d_{x^{2}-y^{2}}$ orbital located at $\mathbf{r}$ to a $d_{x z}$ orbital located at $\mathbf{r} \pm \mathbf{e}_{x}+\mathbf{e}_{z}$ is symmetry allowed. However, there will be a relative sign difference between hopping in the positive and negative $x$ directions. A similar argument applies to hopping between a $d_{x^{2}-y^{2}}$ orbital in the $B$ layer and a $d_{y z}$ orbital in the middle layer. The tunneling between the $B$-layer $d_{x^{2}-y^{2}}$ orbitals and the $d_{x z}$ and $d_{y z}$ orbitals in the middle layer is then

$$
t b_{\mathbf{r} \sigma}^{\dagger}\left[\left(\alpha_{\mathbf{r}-\mathbf{e}_{y}}-\alpha_{\mathbf{r}+\mathbf{e}_{y}}\right)-\left(\beta_{\mathbf{r}-\mathbf{e}_{x}}-\beta_{\mathbf{r}+\mathbf{e}_{x}}\right)\right]_{\sigma}+\text { H.c. },
$$

where $t$ is the hopping strength. The relative sign difference for hopping in the opposite $x(y)$ direction will give rise to the odd-parity elements in the tunneling matrix. Due to the relative $45^{\circ}$ rotation of the $\mathrm{Cu} d_{x^{2}-y^{2}}$ and Ir $d_{x y}$ orbitals, there will always be equal contributions of opposite sign in an overlap integral [35]. The same argument gives a vanishing element for hopping in the straight $\mathbf{e}_{z}$ direction [36].

We recall now that the spin and orbital states of the Ir ion are strongly entangled via intraionic spin-orbit coupling, $H_{\text {SO }}=\lambda \mathbf{L} \cdot \mathbf{S}$, which results in a completely filled $J_{\text {eff }}=3 / 2$ quartet well below the Fermi level, and a half-filled $J_{\text {eff }}=1 / 2$ doublet [17]. This implies that the tunneling process is mostly contributed by half-filled $J_{\text {eff }}=1 / 2$ states, with the following wave functions $[17,37]$ :

$$
\left|f_{\sigma}\right\rangle=\frac{1}{\sqrt{3}}\{\sigma|y z,-\sigma\rangle+i|x z,-\sigma\rangle+|x y, \sigma\rangle\} .
$$

Projection of the Ir $t_{2 g}$ states onto the $f_{\sigma}$ band gives the following correspondence:

$$
\left(\alpha_{\mathbf{r}, \sigma} ; \beta_{\mathbf{r}, \sigma} ; \gamma_{\mathbf{r}, \sigma}\right) \rightarrow \frac{1}{\sqrt{3}}\left(-\sigma f_{\mathbf{r},-\sigma} ; i f_{\mathbf{r},-\sigma} ; f_{\mathbf{r}, \sigma}\right) .
$$

With this substitution in Eq. (7) and after a Fourier transformation, we arrive at the tunneling between the $B$ layer and the middle $(M)$ layer:

$$
H_{B M}=\frac{2}{\sqrt{3}} \sum_{\mathbf{k} \sigma} t\left(\sin k_{x}-i \sigma \sin k_{y}\right) b_{\mathbf{k} \sigma}^{\dagger} f_{\mathbf{k}-\sigma}+\text { H.c. }
$$

We now consider the tunneling between $t_{2 g}$ orbitals in the middle layer and the $d_{x y}$ orbital in the $A$ layer. By arguing as above, we find that there will only be hopping between $d_{x z}$ orbitals at $\mathbf{r}$ and $d_{x y}$ orbitals at $\mathbf{r} \pm \mathbf{e}_{y}+\mathbf{e}_{z}$; we denote this hopping by $t^{\prime}$. Similar arguments apply to the hopping $t^{\prime}$ between $d_{y z}$ orbitals and $d_{x y}$ orbitals. There is also a hopping between the $d_{x y}$ orbitals of the middle and $A$ layer. In this case there is no relative minus sign for hopping in the opposite $x(y)$ direction and hopping to all next-nearest neighbors have the same magnitude, which gives a tunneling of the form $t^{\prime \prime} \gamma_{\mathbf{r} \sigma}^{\dagger}\left(a_{\mathbf{r} \pm \mathbf{e}_{r}}+a_{\mathbf{r} \pm \mathbf{e}_{y}}\right)_{\sigma}+$ H.c. Projecting the above $t^{\prime}$ and $t^{\prime \prime}$ hopping processes onto the $J_{\text {eff }}=1 / 2$ band, we find the tunneling Hamiltonian between the middle layer and the $A$ layer:

$$
\begin{aligned}
H_{M A}= & \frac{2}{\sqrt{3}} \sum_{\mathbf{k} \sigma} f_{\mathbf{k} \sigma}^{\dagger}\left[-i t^{\prime} \sigma\left(\sin k_{x}-i \sigma \sin k_{y}\right) a_{\mathbf{k}-\sigma}\right. \\
& \left.+t^{\prime \prime}\left(\cos k_{x}+\cos k_{y}\right) a_{\mathbf{k} \sigma}\right]+ \text { H.c. }
\end{aligned}
$$

Introducing an effective charge transfer energy $\Delta E$ required to move an electron into the middle layer, we can calculate the effective tunneling Hamiltonian between the $\mathrm{Cu}$ $d_{x^{2}-y^{2}}$ orbitals and the $\mathrm{Ru} d_{x y}$ orbitals to second order in $H_{B M}$ and $H_{M A}$. We then find that the elements of the tunneling matrix (2) are given by

$$
\begin{aligned}
& P_{\mathbf{k}}=i g_{e}\left(\sin ^{2} k_{x}+\sin ^{2} k_{y}\right), \\
& R_{\mathbf{k}}=-g_{o}\left(\cos k_{x}+\cos k_{y}\right)\left(\sin k_{x}-i \sin k_{y}\right),
\end{aligned}
$$

with amplitudes $g_{e}=\frac{4}{3 \Delta E} t t^{\prime}$ and $g_{o}=\frac{4}{3 \Delta E} t t^{\prime \prime}$ in even- and odd-parity channels, correspondingly. While it is difficult to quantify these constants, the above orbital-symmetry considerations confirm that the desired topology of the tunneling matrix, with an opposite parity of the diagonal and offdiagonal elements, is indeed realistic in perovskite-type oxide heterostructures.

Inserting now the tunneling coefficients (11) into Eq. (3), we find a nonvanishing $F_{A B}$ in the $\left(\Delta_{B \mathbf{k}}^{s}, \Gamma_{2}^{-}\right)$and $\left(\Delta_{B \mathbf{k}}^{d}, \Gamma_{4}^{-}\right)$ channels (as it was observed above), and evaluate the corresponding coupling constants $r$ using circular Fermi surfaces for simplicity. The main contribution to $F_{A B}$ (3) stems from the region close to the Fermi surface in the $A$ layer. Away from nesting of the Fermi circles in the $A$ and $B$ layers, the enhancement factor $r$ will be suppressed by $\delta^{2}=\left[\xi_{B}\left(k_{F}^{A}\right)-\xi_{A}\left(k_{F}^{A}\right)\right]^{2}$, where $k_{F}^{A}$ is the Fermi-circle radius in the $A$ layer. An estimate of the enhancement factor $r_{1}$ (due 

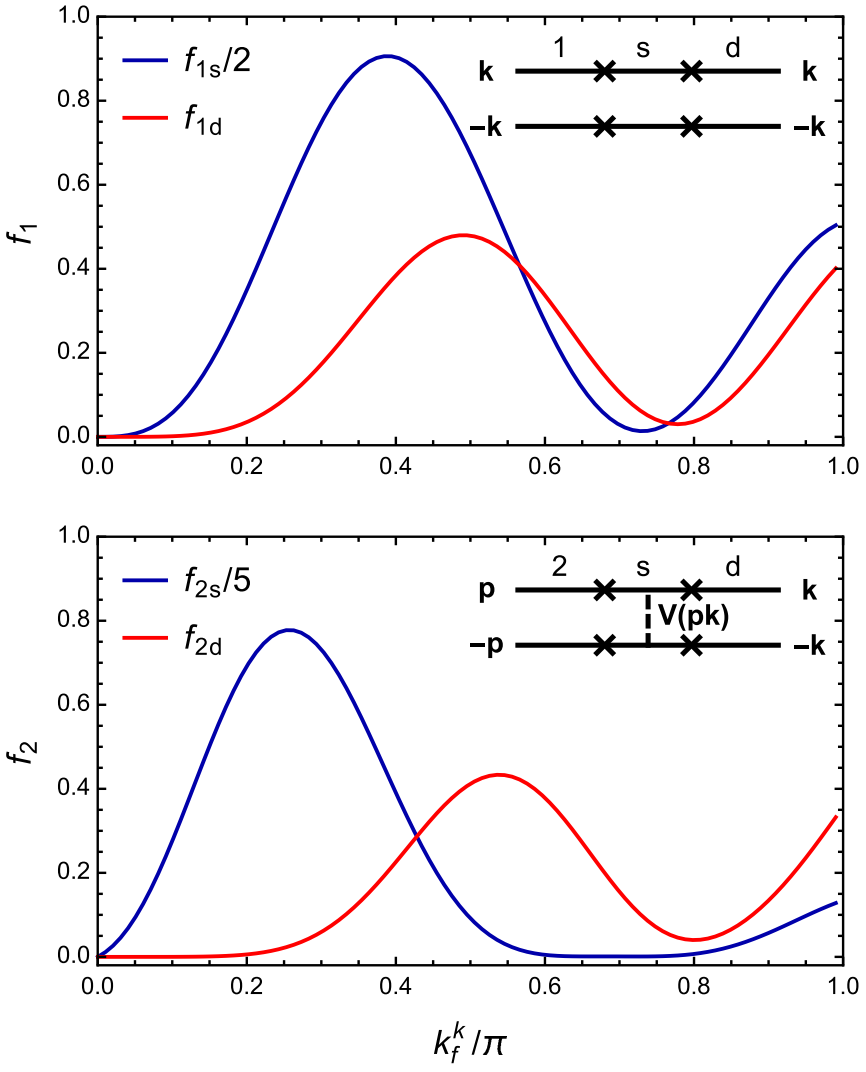

FIG. 3. Dependences of the $f_{1}$ (upper panel) and $f_{2}$ (lower panel) functions [Eqs. (12) and (13)] on the Fermi circle radius in the $A$ layer for the cases of $s$ - and $d$-wave SC in the $B$ layer. Note that the $s$-wave results $f_{1 s}$ and $f_{2 s}$ have been divided by 2 and 5, respectively. The corresponding single- and pair-tunneling processes are shown in the insets.

to single-particle tunneling considered so far) gives

$$
r_{1} \approx \frac{g_{e} g_{o}}{\delta^{2}} \frac{\Delta_{0}}{T_{C 0}} \ln \left(\frac{\delta}{T_{C 0}}\right) f_{1}\left(k_{F}^{A}\right)
$$

where $f_{1}$ is a function that only depends on the Fermi-circle radius. Figure 3 shows $f_{1}\left(k_{F}^{A}\right)$ for the two combinations $\left(\Delta_{B \mathbf{k}}^{s}, \Gamma_{2}^{-}\right)$and $\left(\Delta_{B \mathbf{k}}^{d}, \Gamma_{4}^{-}\right)$. For representative values of $g_{e / o} \sim$ $0.1 \delta, \Delta_{0} / T_{C 0} \sim 30$, and $\Delta_{0} / \delta \sim 0.2$, we find $r_{1} \approx 1.5 f_{1}$.

Another process that contributes to $F_{A B}$ in addition to (3) is the scattering of a Cooper pair of relative momentum $2 \mathbf{p}$ in the
$B$ layer to a pair of relative momentum $2 \mathbf{k}$ in the $A$ layer, due to an electron-electron scattering potential of strength $V$ in the middle layer. This pair-tunneling process, sketched in the lower panel of Fig. 3, is not suppressed by the energy difference $\delta$ and gives the following contribution to the enhancement factor:

$$
r_{2} \approx-\frac{g_{e} g_{o}}{(\Delta E)^{2}} \frac{\Delta_{0}}{T_{C 0}} N_{0} V \ln \left(\frac{2 \omega_{c}}{\Delta_{0}}\right) \ln \left(\frac{\omega_{c}}{T_{C 0}}\right) f_{2}\left(k_{F}^{A}, k_{F}^{B}\right),
$$

where $N_{0}$ is the density of states and $\omega_{c}$ is an upper frequency cutoff. The function $f_{2}$ depends on the Fermi-circle radii in the $A$ and $B$ layers, and is plotted in Fig. 3 as a function of $k_{F}^{A}$ (at $k_{F}^{B}=k_{F}^{A}+\frac{\pi}{10}$ ). Assuming $g_{e / o} \sim 0.1 \Delta E, N_{0}|V| \sim$ 0.5 , and $\omega_{c} / \Delta_{0} \sim 10$, we find $r_{2} \approx \pm 2.5 f_{2}$. Depending on microscopics, $r_{2}$ is positive for an attractive potential (e.g., for a phonon and/or magnetically mediated interaction $V<0$ ), and negative for a repulsive one. From the above estimates, it seems plausible that the single-particle and pair-tunneling processes can give a sizable enhancement factor of the order of $|r| \sim 1$, as used in Fig. 1. In addition, antiferromagnetic (AF) correlations are expected to arise in the iridate layer $[17,38]$. Since pseudospins are spin-orbit composite objects, their AF correlation is in fact a coherent mixture of real-spin singlets and triplets, implying that the iridate AF correlations will further facilitate a coherent singlet-triplet conversion.

In conclusion, we have shown how a coherent coupling between a triplet and a singlet SC can be achieved by means of a time-reversal invariant conversion layer that effectively rotates singlet Cooper pairs into triplets. The conversion is due to tunneling via the strong intraionic spin-orbit coupled states in the middle layer; a possible candidate for such a "pair convertor" might be the iridium oxide $\mathrm{Sr}_{2} \mathrm{IrO}_{4}$. The coherent coupling leads to a dramatic enhancement of the triplet superconductivity, existing well above its "native" critical temperature $T_{C 0}$. Experimentally, the enhanced triplet gap in the quasiparticle spectrum and its temperature dependence as shown in Fig. 1 can be verified using ARPES and STM techniques. The proximity mechanism considered here may also enable the stabilization of topologically nontrivial $p$-wave SCs.

We thank D. Scherer and P. Ostrovsky for helpful discussions. The work is supported by the Research Council of Norway, the Academy of Finland Center of Excellence program, the European Research Council (Grant No. 240362Heattronics), and the Dutch Science Foundation NWO/FOM.
[1] A. P. Mackenzie and Y. Maeno, Rev. Mod. Phys. 75, 657 (2003).

[2] M. Sigrist and K. Ueda, Rev. Mod. Phys. 63, 239 (1991).

[3] F. S. Bergeret, A. F. Volkov, and K. B. Efetov, Phys. Rev. Lett. 86, 4096 (2001).

[4] V. M. Edelstein, Phys. Rev. B. 67, 020505(R) (2003).

[5] M. Eschrig and T. Löfwander, Nat. Phys. 4, 138 (2008).

[6] Y. Tanaka, M. Sato, and N. Nagaosa, J. Phys. Soc. Jpn. 81, 011013 (2012).

[7] F. S. Bergeret and I. V. Tokatly, Phys. Rev. Lett. 110, 117003 (2013).
[8] F. Parhizgar and A. M. Black-Schaffer, Phys. Rev. B 90, 184517 (2014).

[9] A triplet order parameter can also be induced in a different type of system, such as in the vortex phase of high $T_{C}$ SCs [10].

[10] A. G. Lebed, Phys. Rev. Lett. 96, 037002 (2006).

[11] M. Sato, Y. Takahashi, and S. Fujimoto, Phys. Rev. Lett. 103, 020401 (2009).

[12] J. D. Sau, R. M. Lutchyn, S. Tewari, and S. Das Sarma, Phys. Rev. Lett. 104, 040502 (2010). 
[13] S. Nakosai, Y. Tanaka, and N. Nagaosa, Phys. Rev. Lett. 108, 147003 (2012).

[14] S. Takei, B. M. Fregoso, V. Galitski, and S. Das Sarma, Phys. Rev. B 87, 014504 (2013).

[15] Z. Yang, J. Wang, and K. S. Chan, J. Phys.: Condens. Matter 22, 045302 (2010).

[16] P. M. R. Brydon, W. Chen, Y. Asano, and D. Manske, Phys. Rev. B 88, 054509 (2013).

[17] B. J. Kim, H. Ohsumi, T. Komesu, S. Sakai, T. Morita, H. Takagi, and T. Arima, Science 323, 1329 (2009).

[18] Due to breaking of mirror symmetry in the $z$ direction, a Rashbatype spin-orbit coupling can be induced at the $A B$ interface. Except for very special and rare systems [19], this effect is small and therefore ignored here since it will not change the results qualitatively.

[19] M. Diez, A. M. R. V. L. Monteiro, G. Mattoni, E. Cobanera, T. Hyart, E. Mulazimoglu, N. Bovenzi, C. W. J. Beenakker, and A. D. Caviglia, Phys. Rev. Lett. 115, 016803 (2015).

[20] A. Altland and B. Simons, Condensed Matter Field Theory (Cambridge University Press, Cambridge, U.K., 2010).

[21] Higher-order terms in $H_{A B}$ can give rise to a phase-dependent coupling between the two SCs even in the absence of a spin active interface, but these terms will in general be smaller and have a different dependence on the phase difference [22].

[22] C.-K. Lu and S. Yip, Phys. Rev. B 80, 024504 (2009).

[23] M. Sato, Phys. Rev. B 79, 214526 (2009).

[24] X.-L. Qi, T. L. Hughes, and S.-C. Zhang, Phys. Rev. B 81, 134508 (2010).

[25] L. Fu and E. Berg, Phys. Rev. Lett. 105, 097001 (2010).

[26] A. J. Leggett, Rev. Mod. Phys. 47, 331 (1975).
[27] L. Xu, Z. Shu, and S. Wang, Phys. Rev. B 57, 11654 (1998).

[28] A. M. Kadin and A. M. Goldman, Phys. Rev. B 25, 6701 (1982).

[29] H. Y. Hwang, Y. Iwasa, M. Kawasaki, B. Keimer, N. Nagaosa, and Y. Tokura, Nat. Mater. 11, 103 (2012).

[30] The pairing in ruthenates is in reality of multiorbital nature and not yet fully understood (see, e.g., the recent works [31-34]); however, our assumption of the $x y$ orbital is not of a principal importance for illustration of the basic idea of the present Rapid Communication.

[31] C. N. Veenstra, Z.-H. Zhu, M. Raichle, B. M. Ludbrook, A. Nicolaou, B. Slomski, G. Landolt, S. Kittaka, Y. Maeno, J. H. Dil, I. S. Elfimov, M. W. Haverkort, and A. Damascelli, Phys. Rev. Lett. 112, 127002 (2014).

[32] Y. Imai, K. Wakabayashi, and M. Sigrist, Phys. Rev. B 88 144503 (2013).

[33] C. M. Puetter and H.-Y. Kee, Europhys. Lett. 98, 27010 (2012).

[34] T. Scaffidi, J. C. Romers, and S. H. Simon, Phys. Rev. B 89, 220510(R) (2014).

[35] J. C. Slater and G. F. Koster, Phys. Rev. 94, 1498 (1954).

[36] Due to spin-orbit coupling, the $\operatorname{Ir} d_{x^{2}-y^{2}}$ and $d_{x y}$ orbitals mix, and will give an effective hopping between $\mathrm{Cu} d_{x^{2}-y^{2}}$ orbitals in the $B$ layer and the Ir $d_{x y}$ orbitals. There is also a term corresponding to hopping in the straight $\mathbf{e}_{z}$ direction. This effective term will not change the physics and is therefore ignored to keep the model as simple as possible.

[37] G. Jackeli and G. Khaliullin, Phys. Rev. Lett. 102, 017205 (2009).

[38] J. Kim, D. Casa, M. H. Upton, T. Gog, Y.-J. Kim, J. F. Mitchell, M. van Veenendaal, M. Daghofer, J. van den Brink, G. Khaliullin, and B. J. Kim, Phys. Rev. Lett. 108, 177003 (2012). 